TUW-04-20

\title{
Effective Action and Hawking Flux from Covariant Perturbation Theory
}

\author{
D. Hofmann*, W. Kummer ${ }^{\dagger}$, \\ ${ }^{* \dagger}$ Institut für Theoretische Physik \\ Technische Universität Wien \\ Wiedner Hauptstr. 8-10, A-1040 Wien, Austria
}

\begin{abstract}
The computation of the radiation flux related to the Hawking temperature of a Schwarzschild Black Hole or another geometric background is still well-known to be fraught with a number of delicate problems. In spherical reduction, as shown by one of the present authors (W. K.) with D.V. Vassilevich, the correct black body radiation follows when two "basic components" (conformal anomaly and a "dilaton" anomaly) are used as input in the integrated energy-momentum conservation equation. The main new element in the present work is the use of a quite different method, the covariant perturbation theory of Barvinsky and Vilkovisky, to establish directly the full effective action which determines these basic components. In the derivation of $\mathrm{W}$. K. and D.V. Vassilevich the computation of the dilaton anomaly implied one potentially doubtful intermediate step which can be avoided here. Moreover, the present approach also is sensitive to IR (renormalisation) effects. We realize that the effective action naturally leads to expectation values in the Boulware vacuum which, making use of the conservation equation, suffice for the computation of the Hawking flux in other quantum states, in particular for the relevant Unruh state. Thus, a rather comprehensive discussion of the effects of (UV and IR) renormalisation upon radiation flux and energy density is possible.
\end{abstract}

PACS numbers: 4.60.-m, 4.60.Kz, 4.70.Dy

\footnotetext{
${ }^{*}$ E-mail: hofmann@hep.itp.tuwien.ac.at

${ }^{\dagger}$ E-mail: wkummer@tph.tuwien.ac.at
} 


\section{Introduction}

Almost three decades after the (theoretical) discovery of quantum radiation from the event horizon of a geometrical background, in particular from a Black Hole (BH) [1, 2], somewhat surprisingly, still the existence of open problems is an acknowledged phenomenon - even when only large BH-s are considered which, to a good approximation, represent a time-independent curved background.

Actually, the computation of the Hawking effect does not require an analysis of the complete evolution of the (massless) fields between infinitely early and infinitely late times. It is sufficient to exploit the Energy-Momentum (EM) tensor near the future horizon only. The relation between Hawking temperature and the radiation flux at infinity is still the object of some debate. The activity in this field has been rekindled by the work of Bousso and Hawking [3] who, on the basis of a computation in $2 \mathrm{~d}$ dilaton gravity resulting from spherically reducing Einstein gravity [4, claimed that an incoming asymptotic flux and thus "anti-evaporation" occurs. Actually already some time before ref. [3] it had been argued [5] that the so-called conformal anomaly $\langle T\rangle_{2}$, the trace of the EM tensor in two dimensions ${ }^{1}$, which had been the only input considered ${ }^{2}$ in ref. [3], cannot provide the complete answer.

The problem has been focused in refs. [9, 10, 11] where the relation between the missing second piece, the $2 \mathrm{~d}$ "dilaton anomaly" $\left\langle T^{\theta}{ }_{\theta}\right\rangle_{2}$, to the pressure component $\left\langle T^{\theta}{ }_{\theta}\right\rangle_{4}$ of the EM tensor in the original $4 \mathrm{~d}$ theory (in coordinates time, radius and angles $\theta, \varphi)$ has been established. In the following these two essential ingredients of the flux calculation, $\langle T\rangle_{2}$ and $\left\langle T^{\theta}{ }_{\theta}\right\rangle_{2}$ (cf. eqs. (19),(10) below), will be called "basic components" of the EM tensor.

It had been known for a long time [12] that $2 \mathrm{~d}$ minimally coupled massless scalars - i.e. in the absence of a dilaton field - provide the correct flux from the $2 d$ conformal anomaly $\langle T\rangle_{2}$ alone. But an actual computation of the missing piece $\left\langle T^{\theta}{ }_{\theta}\right\rangle_{2}$ was not available until the work of [13].

The determination of $\langle T\rangle_{2}$ precisely fits into the elegant formalism of heat kernel regularisation [14, 15], because in $2 \mathrm{~d}$ "by chance" $\langle T\rangle_{2}$ is simply the trace anomaly of a massless scalar field which is related to the variation of the effective action for a multiplicative (conformal!) factor of the Laplace operator. By contrast, the quantum correction $\left\langle T^{\theta}{ }_{\theta}\right\rangle_{2}$ cannot be computed

\footnotetext{
${ }^{1} \mathrm{~A}$ lower index 2,4 attached to an expectation value means computation in spherically reduced gravity or directly in four dimensions, respectively.

${ }^{2}$ The correct expression for the conformal anomaly in the presence of a $2 d$ dilaton field for spherically reduced gravity [5] and for general $2 d$ dilaton theories [6, 7] taken alone yields the same unphysical flux. For a comprehensive review of general $2 d$ dilaton models we refer to 8 .
} 
as easily in this formalism, because it lacks that key property. For this reason in refs. [13, 16] the Laplace operator, including a general coupling to the dilaton field, has been split into a product of two Dirac operators whose combined determinant need be evaluated in flat space only. Then a (multiplicative!) variation with respect to the dilaton field is observed and allows the computation of the component $\left\langle T^{\theta}\right\rangle_{2}$, dubbed "dilaton anomaly" in that work. Using both basic components in a ("dilaton-deformed") EM conservation equation [9, 11] produced exactly the black body Hawking flux at infinity from $2 \mathrm{~d}$ minimally coupled scalars which would follow from the Hawking temperature computed from, say, the surface gravity at the horizon. The outgoing flux at infinity precisely coincided with the Stefan Boltzmann law for $d=2$, which is related easily to the equally correct $4 \mathrm{~d}$ result. The only further input had been the vanishing of the asymptotic incoming flux together with the condition of finiteness (or at least integrability) of the flux at the horizon (Unruh vacuum) in global Kruskal-Szekeres coordinates. In addition, by direct functional integration an expression for the total effective action in the presence of a general dilaton coupling was obtained. Despite of these satisfactory final results, the step splitting the Laplace operator into two linear Dirac operators from a rigorous mathematical point of view seemed to be a doubtful one.

Therefore, in our present paper we attempt to close that loop hole in an alternative derivation by a new application of the covariant perturbation theory, introduced by Barvinsky and Vilkovisky (BV) [17, 18. This technique allows to proceed directly to the effective action. In ref. [19] it already has been shown that the BV effective action reproduces the correct trace anomaly of conformally coupled scalar fields in $d=4$. Actually, it contains more information than just the trace anomaly, although the latter in general is the only expectation value that can be calculated directly. In particular, the dilaton effective action derived in the present work not only produces the correct $2 \mathrm{~d}$ trace anomaly but also represents another derivation of the dilaton anomaly. However, beside the expression found in [13, 16] we encounter an important IR-renormalisation effect, i.e. something which had been bypassed altogether in the only UV-sensitive previous approach.

That action is used by us only to determine the basic components $\langle T\rangle_{2}$ and $\left\langle T_{\theta}^{\theta}\right\rangle_{2}$, while the remaining components are reconstructed by integration of the EM conservation equation, following the procedure introduced by Christensen and Fulling (CF) 12 .

In Section 2 we present the dilaton model and some characteristic features of spherically reduced (SR) gravity.

Section 3.1 is devoted to the computation of the effective action of the dilaton model by the covariant perturbation theory of refs. [17, 18]. 
In Section 3.2 we discuss the ambiguity of the non-local effective action by a Green function perturbation theory and fix it by appropriate boundary conditions and some infrared regularisation.

The expectation values of the basic components to be derived from the effective action are the subject of Section 4 and the remaining components in the Unruh state are determined by fixing the constants $Q$ and $K$ of ref. 12 accordingly.

In the Conclusions (Section 5) we summarize and discuss the obtained results.

This paper further contains four Appendices: in Appendix A we shortly present the SR procedure and derive some useful formulas. Appendix B contains the second and third order of the Green function perturbation theory. In Appendix $\mathrm{C}$ we show the (non-)conservation of the $2 \mathrm{~d}$ dilaton $\mathrm{EM}$ tensor at the quantum level. In Appendix D the regularisation of the heat kernel is demonstrated.

Important basic calculations and concepts of this paper can be found in more detail in the PhD thesis [20] of one of the authors (D. H.), however a more careful discussion of (UV and IR) renormalisation issues is presented here.

\section{Dilaton Model}

A massless scalar field $S$ is considered on a four-dimensional Schwarzschild spacetime $M$ with coordinates $x^{\mu}=\left(x^{\alpha}, \theta, \varphi\right)$, in 4 d coupled minimally (but not conformally!) to gravity:

$$
L=\int_{M}\left[\frac{c^{2}}{16 \pi G} R^{(4)}+\frac{(\nabla S)^{2}}{2}\right] \sqrt{-g^{(4)}} d^{4} x
$$

In the following we set $c=G=\hbar=k_{B}=1$. This model can be spherically reduced to a dilaton model on a two-dimensional spacetime $L$ by integrating out the isometry coordinates $\theta, \varphi$, (cf. Appendix A (68)):

$$
L_{d i l}=\int_{L}\left\{X R+\frac{(\nabla X)^{2}}{2 X}-2+X\left[\frac{(\nabla S)^{2}}{2}\right]\right\} \sqrt{-g} d^{2} x .
$$

On a four-dimensional spherically symmetric spacetime the EM tensor exhibits only four independent components [12] $\left(T_{2}^{2}=T_{\theta}^{\theta}=T_{\varphi}^{\varphi}\right)$ in a vierbein basis

$$
T_{n}^{m}=\left(\begin{array}{cccc}
T_{0}^{0} & T^{0}{ }_{1} & 0 & 0 \\
-T^{0}{ }_{1} & T^{1}{ }_{1} & 0 & 0 \\
0 & 0 & T^{2}{ }_{2} & 0 \\
0 & 0 & 0 & T^{2}{ }_{2}
\end{array}\right)
$$


where the first block (up to a factor $4 \pi X$ ) equals the two-dimensional EM tensor $T_{\beta}^{\alpha}$ on $L$. Diffeomorphism invariance in the dilaton model (2) on-shell for $S$ implies the $2 \mathrm{~d}$ "non-conservation equation"

$$
\nabla_{\alpha} T_{\beta}^{\alpha}=-\frac{\nabla_{\beta} X}{\sqrt{-g}} \frac{\delta L_{d i l}}{\delta X}
$$

whose solution for the Schwarzschild metric $g_{t t}=-\left(g_{r r}\right)^{-1}=\left(1-\frac{2 M}{r}\right)$

$$
\begin{aligned}
T^{r}{ }_{t}= & -\frac{K}{M^{2}} \\
T_{r}^{r}= & \frac{1}{\left(1-\frac{2 M}{r}\right)}\left\{\frac{Q-K}{M^{2}}\right. \\
& \left.+\int_{2 M}^{r}\left[\frac{M \cdot T}{\left(r^{\prime}\right)^{2}}-\left(1-\frac{2 M}{r^{\prime}}\right) \frac{\partial_{r} X}{\sqrt{-g}} \frac{\delta L_{d i l}^{m}}{\delta X}\right] d r^{\prime}\right\}
\end{aligned}
$$

only depends on the integration constants $Q, K$ and the unknown functions $T, \frac{\delta L_{d i l}^{m}}{\delta X}$ to be identified with the basic components. Eq. (44) is just another expression for the $4 \mathrm{~d}$ conservation equation [12] if one identifies

$$
T_{\theta}^{\theta}=-\frac{1}{4 \pi \sqrt{-g}} \frac{\delta L_{d i l}}{\delta X} .
$$

This relation can be checked easily for the action (21) using the definition of the EM tensor $T_{\mu \nu}=\frac{2}{\sqrt{-g}} \frac{\delta L}{\delta g^{\mu \nu}}$. In Appendix $\mathrm{C}$ we show that in a fixed classical background the non-conservation equation also holds at the level of (renormalized) expectation values,

$$
\nabla^{\beta}\left\langle T_{\alpha \beta}\right\rangle_{r e n}=\frac{\nabla_{\alpha} X}{2}\left\langle m^{2} S^{2}-(\nabla S)^{2}\right\rangle=-\frac{\nabla_{\alpha} X}{\sqrt{-g}} \frac{\delta W}{\delta X},
$$

where $W$ is the generating functional of connected Green functions for the dilaton theory which at the one-loop level coincides with the effective action (when the propagators of the external lines are amputated). At the quantum level the basic components are calculated by variation of the effective action ${ }^{3}$ :

$$
\begin{aligned}
\langle T\rangle_{2} & :=\langle T\rangle=g^{\alpha \beta} \frac{2}{\sqrt{-g}} \frac{\delta W}{\delta g^{\alpha \beta}} \\
\left\langle T^{\theta}{ }_{\theta}\right\rangle_{2} & :=\left\langle T^{\theta}{ }_{\theta}\right\rangle=-\frac{1}{\sqrt{-g}} \frac{\delta W}{\delta X}
\end{aligned}
$$

\footnotetext{
${ }^{3}$ It should be emphasized that $W$ is a two-dimensional action and expectation values derived from it could differ from those calculated in $4 d$.
} 
In the following all quantities are two-dimensional if no dimension index is attached. $T_{t}^{r}$ is the flux component of the EM tensor and differs from the $4 d$ flux (like all components of the EM tensor) by a factor $(4 \pi X)^{-1}$ (the dilaton $X$ is hidden in the spacetime measure $\sqrt{-g_{4}}=X \sqrt{-g_{2}}$ ). The constants $Q, K$ remain to be fixed by the boundary conditions of the fields and are thus related to the quantum state of the system [12].

All physical states are characterized by the choice $Q=0$ which is a necessary condition for the finiteness of the EM tensor at the horizon in global coordinates. The Hartle-Hawking state $|H\rangle$ is given by vanishing total flux $K_{H}=0$ (thermal equilibrium), whereas the Unruh state $|U\rangle$ is determined by vanishing incoming flux (leading to a non-zero $K_{U}$ ). The (unphysical) Boulware state $|B\rangle$ is defined by vanishing fields in the asymptotic region. This is accomplished by setting $K_{B}=0$ and fixing $Q_{B} \neq 0$ appropriately. Although not to be interpreted as a physical state, because of its natural boundary conditions, in a certain sense it nonetheless is the natural state of the effective action. On the one hand, in order to represent a well-defined integral over the fields, natural (vanishing) asymptotic values for them are necessary. On the other hand, in the path integral (cf. (26) below) the field $S^{\prime}$ is a sum of a classical solution $S_{0}$ and a quantum correction $S_{q}$. The standard procedure is to set $S_{0}=0$ - otherwise one would have surface terms that would make the application of the heat kernel method very difficult. This means that the incoming and outgoing states correspond to the vacuum, i.e. the Boulware state. If the expectation value of the EM tensor is calculated from the effective action with the Boulware state values of $K_{B}=0$ and $Q_{B}$, any other quantum state with $K=K_{B}+\tilde{K}, Q=Q_{B}+\tilde{Q}$ can be reconstructed by simply adding (to the first block in (13) ) a term

$$
\left\langle\tilde{T}^{\mu}{ }_{\nu}\right\rangle=\frac{1}{M^{2}}\left(\begin{array}{cc}
\frac{\tilde{K}-\tilde{Q}}{\left(1-\frac{2 M}{r}\right)} & \frac{\tilde{K}}{\left(1-\frac{2 M}{r}\right)^{2}} \\
-\tilde{K} & \frac{\tilde{Q}-\tilde{K}}{\left(1-\frac{2 M}{r}\right)}
\end{array}\right)
$$

which is a special solution of the (non-)conservation equation. Of course, this procedure works only if the basic components are insensitive with respect to the state of the effective action which is true, as long as the radiation does not affect the spacetime geometry significantly, i.e. in the quasi-static phase of a $\mathrm{BH}[20$. 


\section{Non-Local Effective Action}

\subsection{Covariant Perturbation Theory}

The relation [14, 15] between the Euklidean effective action and the heat kernel $e^{-\mathcal{O} \tau}$ for the differential operator $\mathcal{O}$ is given by ${ }^{4}$

$$
W_{\mathcal{E}}[g]=-\left.\frac{1}{2} \frac{d \zeta[s]}{d s}\right|_{s=0}=-\left.\frac{1}{2} \frac{d}{d s} \frac{1}{\Gamma(s)} \int_{0}^{\infty} \frac{d \tau}{\tau^{1-s}} \operatorname{tr} e^{-\mathcal{O} \tau}\right|_{s=0} .
$$

The trace of the heat kernel may be expressed in a coordinate basis

$$
\operatorname{tr} e^{-\mathcal{O} \tau}=\int_{M}\left\langle x\left|e^{-\mathcal{O} \tau}\right| x\right\rangle \sqrt{g} d^{4} x=\int_{M} G_{\mathcal{O}}(x, x ; \tau) \sqrt{g} d^{4} x
$$

In the most common applications the heat kernel is expanded around $\tau=0$ [14, 15. In contrast to that, the aim of the method developed in [17, 18, 19] is to use (12) directly in order to find an expression of the heat kernel which is valid for all values of $\tau$. This allows performing the $\tau$-integration and computing the effective action for any Euklidean Laplacian

$$
\mathcal{O}=-\triangle-E
$$

where $\triangle=g^{\mu \nu} \nabla_{\mu} \nabla_{\nu}$ is the contraction of two general covariant derivatives (which may include a gauge part) by some Euklidean metric $g$, and $E$ is an endomorphism, i.e. some linear bounded map from the space of fields into itself. The covariant perturbation series is based on a separation of the spacetime metric into a flat part $\tilde{g}$ and a perturbing part $h: g_{\mu \nu}=\tilde{g}_{\mu \nu}+h_{\mu \nu}$ (we use the notation of BV). Nevertheless, each order can be represented by covariant expressions corresponding to the full metric $g$, such as the scalar curvature $R$, because the flat metric does not produce gravitational effects $R(\tilde{g})=0$. Then one expands the Laplacian and the heat kernel in orders of $h$ :

$$
\begin{aligned}
\mathcal{O} & =-\triangle_{0}+h^{\mu \nu} \tilde{\nabla}_{\mu} \tilde{\nabla}_{\nu}+\ldots, \\
G_{\mathcal{O}}(\tau) & =e^{-\mathcal{O} \tau}=\sum_{n=0}^{\infty} G_{\mathcal{O}}^{n}(\tau)
\end{aligned}
$$

where $G_{\mathcal{O}}^{0}(\tau)=e^{\tau \triangle_{0}}$ and $\triangle_{0}$ is the flat Laplacian. Up to the second order in the curvature the trace of the heat kernel in even dimensions $d=2 \omega, \omega \in \mathbb{N}$

\footnotetext{
${ }^{4}$ We denote Euklidean objects by an index $\mathcal{E}$.
} 
is found to be ${ }^{5}[18$

$$
\begin{aligned}
& \operatorname{tr} e^{-\mathcal{O} \tau}=\frac{1}{(4 \pi \tau)^{\omega}} \int_{M} \operatorname{tr}\left\{\mathbb{1}+\tau\left(\frac{R}{6}+E\right)\right. \\
& +\tau^{2}\left[R\left(\frac{1}{16(-\tau \triangle)}+\frac{f(-\tau \triangle)}{32}+\frac{f(-\tau \triangle)-1}{8(-\tau \triangle)}+\frac{3[f(-\tau \triangle)-1]}{8(\tau \triangle)^{2}}\right) R\right. \\
& \left.\left.+E\left(\frac{f(-\tau \triangle)}{6}+\frac{f(-\tau \triangle)-1}{2(-\tau \triangle)}\right) R+R \frac{f(-\tau \triangle)}{12} E+E \frac{f(-\tau \triangle)}{2} E\right]\right\} \sqrt{g} d^{2 \omega} x
\end{aligned}
$$

where

$$
f(x)=\int_{0}^{1} e^{-a(1-a) x} d a .
$$

In the present paper we restrict ourselves to the second order of covariant perturbation theory, i.e. terms up to $R^{2}, E R, E^{2}$. This is at least sufficient to compute the exact trace anomaly which is completely determined by a single term of the local Seeley-DeWitt expansion [19, 15] corresponding to that order. With respect to other expectation values like the dilaton anomaly the necessity for higher orders cannot be excluded, a priori, though.

For $d=2$ the trace of the heat kernel (17) (to this order) in (12) produces five types of integrals. They contain IR or (and) UV divergences that have to be regularized by restricting the range of the $\tau$-integration as $\int_{\varepsilon}^{T} d \tau$ in the limit $T \rightarrow \infty, \varepsilon \rightarrow 0+$. Examples for these rather tedious calculations are shown in Appendix D. They can be done analytically to leading order in $T, \varepsilon$ for these IR, resp. UV regularisation parameters, and for the corresponding next finite terms. The formal contribution of these terms to the effective action (12) is given by

$$
\begin{aligned}
\left.\frac{d}{d s}\left\{\frac{1}{\Gamma(s)} \int_{0}^{\infty} \tau^{s-1} d \tau\right\}\right|_{s=0} & =\ln \frac{T}{\varepsilon} \quad \text { IR } U V(19) \\
\left.\frac{d}{d s}\left\{\frac{1}{\Gamma(s)} \int_{0}^{\infty} \tau^{s-2} d \tau\right\}\right|_{s=0} & =\frac{1}{\varepsilon} \quad U V(20) \\
\left.\frac{d}{d s}\left\{\frac{1}{\Gamma(s)} \int_{0}^{\infty} \tau^{s} f(-\tau \triangle) d \tau\right\}\right|_{s=0} & =\frac{2 \cdot\left[\ln (-T \triangle)+\gamma_{E}\right]}{-\triangle} I R(21) \\
\left.\frac{d}{d s}\left\{\frac{1}{\Gamma(s)} \int_{0}^{\infty} \tau^{s-1} \frac{f(-\tau \triangle)-1}{-\triangle} d \tau\right\}\right|_{s=0} & =\frac{2-\ln (-T \triangle)-\gamma_{E}}{-\triangle R(22)} \\
\left.\frac{d}{d s}\left\{\frac{1}{\Gamma(s)} \int_{0}^{\infty} \tau^{s-2} \frac{f(-\tau \triangle)-1}{\triangle^{2}} d \tau\right\}\right|_{s=0} & =\frac{\ln (-T \triangle)+\gamma_{E}-\frac{8}{3}}{6(-\triangle)} U V(23)
\end{aligned}
$$

\footnotetext{
${ }^{5}$ In the following we set $\omega=1$, i.e. $d=2$.
} 
where $\gamma_{E} \approx 0,57721$ is the Euler constant. Collecting all terms in the heat kernel (17) it turns out that the contributions from the divergent parts $\varepsilon^{-1}, \ln \varepsilon$, and $\ln T$ surprisingly cancel to zero in those nonlocal terms containing the scalar curvature $R$. Thus, the most general two-dimensional regularized effective action to second order of perturbation theory in $R$, resp. $E$ reads

$W_{\mathcal{E}}^{r e g}[g]=\frac{1}{96 \pi} \int_{L}\left[12 c_{0}-c_{1}(2 R+12 E)+(R+12 E) \frac{1}{\triangle} R+12 E \frac{c_{2}}{\triangle} E\right] \sqrt{g} d^{2} x$.

Here we have introduced the following regularisation terms: $c_{0}=\varepsilon^{-1}, c_{1}=$ $\ln (\varepsilon / T), c_{2}=\ln (-T \triangle)+\gamma_{E}$. Actually the term $\propto c_{1} R$ can be dropped, being a total divergence. Note that $c_{2}$ beside a logarithmic divergence contains an ill-defined expression in the Laplacian. In Section 4 the (eventual) contributions of the regular and divergent terms will be discussed separately.

\subsection{Effective Dilaton Action}

So far all steps in this section were valid for general Laplacian $\triangle$ and endomorphism $E$. In order to establish the effective action of the dilaton model (2) we have to specify $\triangle$ and $E$ accordingly, while returning to Lorentzian spacetime. To achieve this we must reconsider the four-dimensional generating functional, determined by the matter part of (11)

$$
Z\left[g_{4}\right]=\mathcal{N} \int \mathcal{D}\left(\sqrt[4]{-g_{4}} S\right) \cdot e^{i L_{m}^{4}\left[g_{4}, S\right]}=\mathcal{N} \int \mathcal{D}\left(\sqrt[4]{-g_{4}} S\right) \cdot e^{-i \int_{M} S \square S \sqrt{-g_{4}} d^{4} x}
$$

$\mathcal{N}$ is a normalization constant and the factor $\sqrt[4]{-g}$ in the path integral measure establishes diffeomorphism invariance [21] of the path integral. The SR generating functional is obtained by introducing the SR d'Alembertian $\square_{4}=\square_{2}+\frac{\nabla X}{X} \nabla$ (cf. (69) in the Appendix for $d=4$ ) and measure $\sqrt{-g_{4}}=X \sqrt{-g_{2}}$ in the classical action and by integration over the angular coordinates $\theta, \varphi$ :

$$
Z\left[g_{2}\right]=\mathcal{N} \int \mathcal{D}\left(\sqrt[4]{-g_{2}} \sqrt{X} S\right) \cdot e^{-4 \pi i \int_{L} S\left(X \square_{2}+\nabla X \nabla\right) S \sqrt{-g_{2}} d^{2} x}
$$

It is now convenient to define a new field $S^{\prime}:=\sqrt{X} S$ such that ${ }^{6} L_{m}\left[g_{2}, S^{\prime}\right]=$ $-\int_{L} S^{\prime} \mathcal{O}_{\mathcal{M}} S^{\prime} \sqrt{-g_{2}} d^{2} x$ defining the complete d'Alembertian of the dilaton

\footnotetext{
${ }^{6}$ In [20] the original field $S$ was preserved, leading to an additional dilaton factor $X$ from the measure during spherical reduction. In the $2 \mathrm{~d}$ action this difference can be described by a conformal transformation of the metric by this factor, not affecting the Hawking flux but other components of the EM tensor.
} 
model $^{7}$

$$
\begin{aligned}
\mathcal{O}_{\mathcal{M}} & =\frac{1}{\sqrt{X}}\left(X \square_{2}+\nabla X \nabla\right) \frac{1}{\sqrt{X}}=\square_{2}+E_{\mathcal{M}}, \\
E_{\mathcal{M}} & =\frac{(\nabla X)^{2}}{4 X^{2}}-\frac{\square X}{2 X} .
\end{aligned}
$$

Inserting these results into (24) and going back to Lorentzian spacetime $d \tau=$ $i d t, W_{\mathcal{M}}=i W_{\mathcal{E}}, \triangle \rightarrow-\square, R_{\mathcal{E}} \rightarrow-R_{\mathcal{M}}, E_{\mathcal{E}} \rightarrow-E_{\mathcal{M}}$ the effective action of the dilaton model (2) follows ${ }^{8}$ :

$$
\begin{aligned}
& W_{\mathcal{M}}^{d i l}[g]=\frac{1}{96 \pi} \int_{L}\left\{-12 c_{0}-3 c_{1}\left[\left(\frac{\nabla X}{X}\right)^{2}-2 \frac{\square X}{X}\right]\right. \\
& +\left[R+3\left(\frac{\nabla X}{X}\right)^{2}-6 \frac{\square X}{X}\right] \frac{1}{\square} R \\
& \left.+\frac{3}{4}\left[\left(\frac{\nabla X}{X}\right)^{2}-2 \frac{\square X}{X}\right] \frac{c_{2}}{\square}\left[\left(\frac{\nabla X}{X}\right)^{2}-2 \frac{\square X}{X}\right]\right\} \sqrt{-g} d^{2} x \text {. }
\end{aligned}
$$

A particular, attractive feature of the $2 \mathrm{~d}$ dilaton model is that most of its effective action (29) can be brought into a local form by choosing a conformal gauge $g_{\alpha \beta}=e^{2 \rho} \eta_{\alpha \beta}$ of the spacetime metric. The scalar curvature then becomes $R=-2 \square \rho$ and the endomorphism $E=\square \phi-(\nabla \phi)^{2}$. It is convenient to represent the dilaton field in the form $X=e^{-2 \phi}$. If one (naively) uses the relation

$$
\square^{-1} \square=1
$$

one obtains the local part of the effective dilaton action

$$
W_{l}[g]=\frac{1}{24 \pi} \int_{L}\left\{-3 c_{0}+3 c_{1}(\nabla \phi)^{2}+\rho \square \rho+6 \rho(\nabla \phi)^{2}-6 \rho \square \phi\right\} \sqrt{-g} d^{2} x
$$

which turns out to be identical to the one derived in [13. However a new contribution

$$
W_{n l}[g]=\frac{1}{24 \pi} \int_{L}\left\{3\left[\square \phi-(\nabla \phi)^{2}\right] \frac{c_{2}}{\square}\left[\square \phi-(\nabla \phi)^{2}\right]\right\} \sqrt{-g} d^{2} x
$$

appears in our present approach which cannot be brought into a local form. Nevertheless, we will be able to show below that the relevant expectation

\footnotetext{
${ }^{7} \mathcal{M}$ indicates Minkowski signature.

${ }^{8}$ In the following we omit the index dil.
} 
value derived from (32) is local after all but ill-defined. It is remarkable that the divergent terms do not contain the scalar curvature.

Obviously, the form (31) is not unique. Namely, by naively using relation (30) one implicitly disregards a homogeneous solution $\chi$ of the wave equation $\square \chi=0$. It is the aim of the next section to present a heuristic argument that the proper choice is indeed $\chi=0$, and thus (301) is in agreement with the boundary conditions which we should impose onto the scalar field $S$.

\subsection{Homogeneous Solution and Boundary Conditions}

The relation of a particular choice of $\chi$ to the boundary conditions of the (massless) scalar field $S$ can be seen when writing the inverse d'Alembertian as an integral over the Green function of $S$ and applying Green's theorem whereby $\square G\left(x, x^{\prime}\right)=-\delta\left(x-x^{\prime}\right)$ and $\square f=F$ :

$$
\begin{gathered}
-\frac{1}{\square} F=\int_{L} G\left(x, x^{\prime}\right) F\left(x^{\prime}\right) \sqrt{-g^{\prime}} d^{2} x^{\prime} \\
=-f(x)-\oint_{\partial L}\left[f \nabla_{\alpha}^{\prime} G-G \nabla_{\alpha}^{\prime} f\right] \sqrt{-g^{\prime} \varepsilon^{\alpha}}{ }_{\beta}\left(d x^{\prime}\right)^{\beta} \\
\quad=-f(x)-\left.\int_{2 M}^{\infty} f\left(x^{\prime}\right) \partial_{t^{\prime}} G \frac{d r^{\prime}}{\left(1-\frac{2 M}{r^{\prime}}\right)}\right|_{t^{\prime}=\infty}+\left.\ldots\right|_{t^{\prime}=-\infty} \\
-\left.\int_{-\infty}^{\infty}\left[f\left(x^{\prime}\right) \partial_{r^{\prime}} G-G \partial_{r^{\prime}} f\left(x^{\prime}\right)\right]\left(1-\frac{2 M}{r^{\prime}}\right) d t^{\prime}\right|_{r^{\prime}=2 M}+\left.\ldots\right|_{r^{\prime}=\infty} .
\end{gathered}
$$

In the step to the third line the explicit form of the Schwarzschild metric has been used. According to (33), to guarantee regularity of the boundary terms, $G\left(x, x^{\prime}\right)$ must vanish at least linearly for $r^{\prime}=2 M$. This is a natural condition for the Green functions since the manifold $L$ is in fact a half-plane that is bounded by the coordinate singularity $r=2 M$. Further, one must employ some infrared regularisation to render finite the support of the Green functions. As the flux is measured at some finite distance $r$ from the $\mathrm{BH}$ at some instant of time $t$ during the quasi-static phase all boundary terms should vanish after that infrared regularisation. This can be realized by simply dropping all boundary terms ${ }^{9}$. But then (33) reduces to $-\frac{1}{\square} F=-f$ (provided that $f$ has at most logarithmic divergences on the horizon) and relation (30) is fulfilled.

It is instructive to analyze expressions like $\square^{-1}$ more explicitly. Unfortunately, the Green functions on a Schwarzschild spacetime cannot be obtained

\footnotetext{
${ }^{9}$ Alternatively one could introduce a finite mass parameter or consider a manifold of finite size.
} 
in a closed form. Nevertheless, one may construct a heuristic argument by considering the properties of a formal perturbation series

$$
\begin{aligned}
& G\left(x, x^{\prime}\right)=G_{0}\left(x, x^{\prime}\right)+\int_{L}^{\prime \prime} G_{0}\left(x, x^{\prime \prime}\right) \delta \square^{\prime \prime} G_{0}\left(x^{\prime \prime}, x^{\prime}\right) d^{2} x^{\prime \prime} \\
& \quad+\int_{L}^{\prime \prime} \int_{L}^{\prime \prime \prime} G_{0}\left(x, x^{\prime \prime}\right) \delta \square^{\prime \prime} G_{0}\left(x^{\prime \prime}, x^{\prime \prime \prime}\right) \delta \square^{\prime \prime \prime} G_{0}\left(x^{\prime \prime \prime}, x^{\prime}\right) d^{2} x^{\prime \prime} d^{2} x^{\prime \prime \prime}+\ldots,
\end{aligned}
$$

where

$$
\square=\left(1-\frac{2 M}{r}\right)^{-1} \partial_{t}^{2}-\partial_{r}\left[\left(1-\frac{2 M}{r}\right) \partial_{r}\right]
$$

is the two-dimensional Schwarzschild d'Alembertian and

$$
\delta \square=\square-\square_{0}=\frac{2 M}{r-2 M} \partial_{t}^{2}+\partial_{r}\left(\frac{2 M}{r} \partial_{r}\right)
$$

is the difference to $\square_{0}$, the flat one.

In eqs. (74), resp. (76) of Appendix B the results for the second, resp. the third order of the perturbation series are given.

The Green functions on the flat half-plane which fulfill the imposed boundary conditions are easy to construct, singling out the appropriate eigenfunctions. For instance, the flat retarded Green function reads

$$
\begin{aligned}
G_{0}^{r e t}= & \theta\left(t-t^{\prime}\right) G_{0}^{(0)} \\
G_{0}^{(0)}= & \frac{1}{2}\left[\theta\left(r-r^{\prime}+t^{\prime}-t\right)-\theta\left(r-r^{\prime}+t-t^{\prime}\right)\right. \\
& \left.-\theta\left(r+r^{\prime}-4 M+t^{\prime}-t\right)+\theta\left(r+r^{\prime}-4 M+t-t^{\prime}\right)\right] .
\end{aligned}
$$

In the same manner the flat advanced and Feynman-type "causal" Green functions can be obtained. It should be noted that the non-local terms in the effective action, having the form $\int_{L} G\left(x, x^{\prime}\right) F\left(x^{\prime}\right) \sqrt{-g} d^{2} x^{\prime}$ with timeindependent $F=F(r)$, are independent of the type of Green function as can be verified easily. Thus only the retarded Green function (37) need to be considered and any further ambiguity of that type disappears.

Among the non-local expressions that appear in the effective action (29) the first one has the form $\square^{-1} R$. The first three orders of the perturbation series with (34) and the formulas (74), (176) of Appendix B yield

$$
\begin{aligned}
& \int_{L} G\left(x, x^{\prime}\right) R\left(x^{\prime}\right) \sqrt{-g^{\prime}} d^{2} x^{\prime}=1+ \frac{1}{2}+\frac{1}{3}+\cdots-\frac{2 M}{r}-\frac{2 M^{2}}{r^{2}}-\frac{8 M^{3}}{3 r^{3}}-\ldots \\
& \rightarrow \ln \left(1-\frac{2 M}{r}\right)-\ln 0=2 \rho-\ln \tilde{\varepsilon}, \quad(39)
\end{aligned}
$$


$\tilde{\varepsilon} \rightarrow 0$, which is suggested by the formal summation in the first line. Bearing in mind that $R=-2 \square \rho=-\square \ln \left(1-\frac{2 M}{r}\right)$ we may argue that the perturbation series produces a homogeneous solution which is just an infinite constant $\chi_{\rho}=-\frac{1}{2} \ln \tilde{\varepsilon}$. Its appearance could have been expected also as it is "required" to shift the absolute value of the integral (39) in accordance with the boundary conditions: if one inserts $r=2 M$ on the l.h.s. the value of the integral must become zero. The action of the inverse d'Alembertian on the half-plane on a function $F=\square f$ thus becomes

$$
\square^{-1} \square f(r)=f(r)-f(2 M) .
$$

But this is consistent with the proper definition of a delta-function on the half-plane:

$$
\delta^{h p}\left(x-x^{\prime}\right):=\delta\left(r-r^{\prime}\right) \delta\left(t-t^{\prime}\right)-\delta\left(r+r^{\prime}-4 M\right) \delta\left(t-t^{\prime}\right)
$$

Using $\delta^{h p}$ in Green's theorem (33) immediately yields indeed (40). In any case this boundary term does not change the effective action (31) because all homogeneous constants can be absorbed anyway by the renormalisation constant $c_{1}$, which, on the other hand, has no effect on the Hawking flux (c.f. (53) below).

Finally, the first order of the second type of non-local term $\square^{-1} \square \phi=$ $\square^{-1}\left(\frac{4 M-r}{r^{3}}\right)$ in the effective action should be checked:

$$
\int_{L} G_{0}\left(x, x^{\prime}\right) \frac{4 M-r^{\prime}}{\left(r^{\prime}\right)^{3}} d^{2} x^{\prime} \approx \ln \left(\frac{r}{2 M}\right)-\left(1-\frac{2 M}{r}\right)+\ldots
$$

The leading order $\ln \left(\frac{r}{2 M}\right)$ corresponds to the expected result as $\phi=-\ln r$. To this order nothing can be said about the additional term $-\left(1-\frac{2 M}{r}\right)$ which should be absorbed by higher orders of the perturbation series.

\section{Hawking Flux}

\subsection{Regular Part}

Having derived in Section 2 the effective action of the dilaton model after fixing its local form (31) by considering boundary conditions, it is now straightforward to calculate the expectation values of the basic components by functional variation for $\rho$ and $X$ (or, more conveniently, $\phi$ ). First we only

regard the regular part $\propto \int_{L}\left[\rho \square \rho+6 \rho\left(\nabla \phi^{2}\right)-6 \rho \square \phi\right] \sqrt{-g} d^{2} x$ of (31). The trace (9) then becomes

$$
\langle T\rangle=-\frac{1}{\sqrt{-g}} \frac{\delta W_{r e g}}{\delta \rho}=\frac{M}{3 \pi r^{3}},
$$


and the pressure component (10), remembering that $X=e^{-2 \phi}$,

$$
\left\langle T^{\theta}{ }_{\theta}\right\rangle=\frac{1}{2 X \sqrt{-g}} \frac{\delta W_{\text {reg }}}{\delta \phi}=\frac{-1}{8 \pi r^{5}}\left\{4 M+(4 M-r)\left[\ln \left(1-\frac{2 M}{r}\right)-\ln \tilde{\varepsilon}\right]\right\} .
$$

The appearance of an infinite constant $\ln \tilde{\varepsilon}$ can be traced back to the particular boundary conditions used in this work to define the inverse d'Alembertian (40). It can be shifted to the (also infinite) regularisation constant $c_{1}$, see next Section ${ }^{10}$.

As discussed in the Introduction, the basic components can be considered as being state-independent, whereas the proper quantum state for a well-defined effective action has been argued to be the static (unphysical) Boulware state. Thus we must rely on the conservation equation (8) to compute the remaining components of the EM tensor in the Unruh state. A necessary condition for regularity of the EM tensor at the horizon was $Q_{U}=0$. The other constant $K_{U}$ is determined by the condition that the incoming flux vanishes

$$
\begin{gathered}
\left\langle U\left|T_{++}\right| U\right\rangle=\frac{\left(1-\frac{2 M}{r}\right)}{4}\left(\left\langle T^{t}{ }_{t}\right\rangle-\left\langle T^{r_{*}}{ }_{r_{*}}\right\rangle-2\left\langle T^{r_{*}}{ }_{t}\right\rangle\right) \\
=\frac{\left(1-\frac{2 M}{r}\right)}{4}\left(\langle T\rangle-2\left\langle T_{r}^{r}\right\rangle-2\left\langle T^{r_{*}}{ }_{t}\right\rangle\right) \\
\stackrel{r \rightarrow \infty}{\rightarrow} \frac{K_{U}}{M^{2}}-\frac{\int_{2 M}^{\infty}\left[\frac{M}{\left(r^{\prime}\right)^{2}}\langle T\rangle+\left(1-\frac{2 M}{r^{\prime}}\right) \partial_{r} X\left\langle T^{\theta}{ }_{\theta}\right\rangle\right] d r^{\prime}}{2}-\frac{Q_{U}}{2 M^{2}}=0 .
\end{gathered}
$$

Inserting (43), (44) and fixing the dilaton field to its standard form $X=r^{2}$ in spherical coordinates we obtain

$$
K_{U}=M^{2} \int_{2 M}^{\infty}\left[\frac{M}{2\left(r^{\prime}\right)^{2}}\langle T\rangle_{2}+\left(r^{\prime}-2 M\right)\left\langle T_{\theta}^{\theta}\right\rangle_{2}\right] d r^{\prime}=-\frac{1}{768 \pi} .
$$

By the CF equations (5), (6) the total flux $F_{\text {reg }}$ from the regular part of the effective action through a large spherical shell surrounding the $\mathrm{BH}$ is then given by

$$
F_{\text {reg }}=\left\langle U\left|T^{r}{ }_{t}\right| U\right\rangle_{2}=-\frac{K_{U}}{M^{2}}=\frac{1}{768 \pi M^{2}},
$$

which precisely coincides with the result obtained in refs. [13, 16]. The related flux in $4 \mathrm{~d}$ through a sphere of size $4 \pi r^{2}$ becomes

$$
\left\langle U\left|T^{r}{ }_{t}\right| U\right\rangle_{4}=\frac{1}{3072 \pi^{2} M^{2} r^{2}} .
$$

\footnotetext{
${ }^{10}$ For the moment we keep this notation until we consider the contribution of the corresponding divergent term in the effective action.
} 
As a consequence the $\mathrm{BH}$ behaves as a black body at Hawking temperature with the radiation flux according to Stefan-Boltzmann's law [13, 16]. It is appropriate, though, to emphasize at this point the drawback of this solution to the energy flow problem [10]: at the horizon the energy density and other components of the EM tensor exhibit a logarithmic singularity in global coordinates! As argued in [16 this singularity (being integrable) is not in contradiction with the finiteness of the total flux. Actually, we also obtain the outgoing flux in light-cone coordinates $T_{--}$with that singularity (eq. (97) of [16]) in our present approach

$\left\langle U\left|T_{--}\right| U\right\rangle=\frac{\left(1-\frac{2 M}{r}\right)^{2}}{768 \pi M^{2} r^{2}}\left\{r^{2}+4 M r+12 M^{2}+48\left[\ln \left(1-\frac{2 M}{r}\right)-\ln \tilde{\varepsilon}\right]\right\}$.

\subsection{Divergent Terms}

The first divergent term $\propto c_{0}=\varepsilon^{-1}$ in (31) is a pure UV divergence and has the form of a cosmological constant:

$$
W_{c_{0}}=\frac{-1}{8 \pi} \int_{L} c_{0} \sqrt{-g} d^{2} x=\frac{-c_{0}}{8 \pi} \int_{L} e^{2 \rho} d^{2} x
$$

It contributes to the trace of the EM tensor $\langle T\rangle_{c_{0}}=\frac{c_{0}}{4 \pi}$ and hence also to the asymptotic flux ( $\varepsilon$ has dimension $M^{2}$ )

$$
K_{U}^{c_{0}}=M^{2} \int_{2 M}^{\infty} \frac{M\langle T\rangle_{c_{0}}}{2 r^{2}} d r=\frac{M^{2} c_{0}}{16 \pi} .
$$

This divergent contribution to the EM tensor can be interpreted as an infinite vacuum energy because it even appears in the case of flat spacetime $M=$ $R=0$. A renormalized EM tensor can thus be defined by subtracting the flat spacetime value (with flat metric $\eta)\left\langle T_{\alpha \beta}\right\rangle_{r e n}:=\left\langle T_{\alpha \beta}\right\rangle_{g_{L}}-\left\langle T_{\alpha \beta}\right\rangle_{\eta}$ or by simply setting $c_{0}=0$.

Further, we had an IR-UV divergence $\propto c_{1}=\ln (\varepsilon / T)$ in (31), contributing only to the pressure component

$$
\left\langle T^{\theta}{ }_{\theta}\right\rangle_{c_{1}}=\frac{c_{1}(r-4 M)}{8 \pi r^{5}} .
$$

Although the asymptotic behavior of the EM tensor is unaffected by $c_{1}$ because

$$
K_{U}^{c_{1}}=M^{2} \int_{2 M}^{\infty}(r-2 M)\left\langle T^{\theta}{ }_{\theta}\right\rangle_{c_{1}} d r=0
$$


it produces infinite contributions to the EM tensor in higher orders in $r$. Comparing with (44) we observe that $c_{1}$ appears in the same place as the homogeneous solution $\chi_{\rho}=-\frac{1}{2} \ln \tilde{\varepsilon}$ needed to fulfill the boundary conditions (cf. Section 3.3) by (40). After all, any constant homogeneous solution $\chi_{\rho}$ leaves the asymptotic flux invariant and can be shifted to the regularisation constant $c_{1}$.

The situation clearly is different if the homogeneous solution is a function. If, for instance, one chooses as in ref. [10]

$$
\tilde{\chi}_{\rho}=\frac{1}{2}\left[\frac{r}{2 M}-1+\ln \left(\frac{r}{2 M}-1\right)-\ln \tilde{\varepsilon}\right],
$$

in order to eliminate the logarithmic singularity of the flux at the horizon, this would mean that the corresponding non-local term in the effective action had the form (cf. (39))

$$
\int_{L} G\left(x, x^{\prime}\right) R\left(r^{\prime}\right) d^{2} x^{\prime}=\frac{r}{2 M}-1+\ln \left[\left(\frac{r}{2 M}-1\right)\left(1-\frac{2 M}{r}\right)\right]-2 \ln \tilde{\varepsilon}
$$

and the asymptotic Hawking flux not only is affected, but even would become negative because $\tilde{K}_{U}>0$ :

$$
\tilde{K}_{U}=K_{U}+M^{2} \int_{2 M}^{\infty} \frac{\left(r^{\prime}-2 M\right)\left(r^{\prime}-4 M\right)}{4 \pi\left(r^{\prime}\right)^{5}} \tilde{\chi}_{\rho} d r^{\prime}=K_{U}+\frac{1}{128 \pi}=\frac{5}{768 \pi}
$$

This is the result obtained in ref. [10], eq. (16). Also the other components of the EM tensor calculated with $\tilde{\chi}_{\rho}$ in (54) can be verified to agree with those of that work. As observed by the authors of [10] themselves, then the weak energy condition is clearly violated in the asymptotic region. We do not believe that this serious consequence of the choice (54) justifies its aim to eliminate the logarithmic singularity at the horizon [10].

Finally, we have a non-local divergent part (32) of the effective action $\propto c_{2}$ whose contribution to the basic components can be localized in a conformal gauge

$$
\begin{aligned}
\langle T\rangle_{c_{2}} & =-\frac{1}{\sqrt{-g}} \frac{\delta W_{n l}}{\delta \rho}=-\frac{1}{8 \pi \sqrt{-g}} \frac{\delta}{\delta \rho} \int_{L} E \frac{\ln \left(-T e^{-2 \rho} \square_{0}\right)}{\square} E \sqrt{-g} d^{2} x \\
& =\frac{1}{4 \pi} E \frac{1}{\square} E=\frac{\square \rho[\rho-\rho(2 M)]}{4 \pi}=\frac{M\left[\ln \left(1-\frac{2 M}{r}\right)-\ln \tilde{\varepsilon}\right]}{4 \pi r^{3}} \\
\left\langle T^{\theta}{ }_{\theta}\right\rangle_{c_{2}} & =\frac{1}{2 X \sqrt{-g}} \frac{\delta W_{n l}}{\delta \phi}=\frac{1}{8 \pi r^{2} \sqrt{-g}} \int_{L} \frac{\delta E}{\delta \phi} \frac{c_{2}}{\square} E \sqrt{-g} d^{2} x \\
& =\frac{[\square+2 \square \phi+2 \nabla \phi \nabla]}{8 \pi r^{2}} \frac{c_{2}}{\square} E=\frac{[\square+2 \square \phi+2 \nabla \phi \nabla] c_{2}[\rho-\rho(2 M)]}{8 \pi r^{2}},
\end{aligned}
$$


where we have used the relation (after having varied the effective action!)

$$
E=\square \phi-(\nabla \phi)^{2}=\frac{4 M-r}{r^{3}}-\frac{2 M-r}{r^{3}}=\frac{2 M}{r^{3}}=-\frac{R}{2}=\square \rho .
$$

(58) contains an ill-defined expression $\ln (T \square)$ that cannot be treated further. Even if $c_{2}$ were only a constant, the pressure component would change by a term $\left\langle T^{\theta}{ }_{\theta}\right\rangle_{c_{2}}=\frac{c_{2}}{8 \pi r^{5}}\left\{4 M+(4 M-r)\left[\ln \left(1-\frac{2 M}{r}\right)-\ln \tilde{\varepsilon}\right]\right\}$ identical to the original expression (44). In that case the asymptotic flux would be affected too: $F_{\text {reg }} \rightarrow F_{\text {reg }} \cdot\left(1-3 c_{2}\right)$. Because $F_{\text {reg }}$ is supposed to be the correct result this constant had to be zero.

The appearance of IR divergences within the covariant perturbation theory (in contrast to the local Seeley-DeWitt expansion used in former derivations [13, 16]) is not surprising as it allows for infinitely large values of the eigentime $\tau$, corresponding to zero modes. As long as a more detailed analysis of these terms does not exist we can only highlight the existence of such terms while assuming that their (infinite) contribution to the EM tensor might be canceled by some mechanism that has not been considered yet and may necessitate the inclusion of (even arbitrary ?) higher orders in covariant perturbation theory.

\section{Conclusions and Outlook}

The Hawking flux from a spherical Black Hole has been reconsidered, whereby we followed the line of solving the EM conservation law, as proposed by Christensen and Fulling many years ago [12]. We have linked the formalism of $d=4$ to the "non-conservation equation" in the effective $d=2$ dilaton theory where as in $4 d$ gravity, beside two constants, only two "basic components" are a necessary input, the $2 \mathrm{~d}$ trace anomaly $\langle T\rangle_{2}$ of the EM tensor and the "dilaton anomaly" $\left\langle T^{\theta}\right\rangle_{2}$ to be interpreted also as (part of) the pressure component $\left\langle T^{\theta}{ }_{\theta}\right\rangle_{4}=\left\langle T^{\varphi}\right\rangle_{4}$ in $d=4$.

Whereas the computation of $\langle T\rangle_{2}$ is known for a long time to fit perfectly into the heat kernel formalism, in its single previous determination of refs. [13, 16. $\left\langle T^{\theta}{ }_{\theta}\right\rangle_{2}$ only by a tour de force argument had been made accessible to that technique. In our present work we replace both derivations by a new application of the "covariant perturbation theory", introduced by Barvinsky and Vilkovisky [17, 18, which allows the direct determination of the effective action also in the presence of dilaton fields. Again the heat kernel is used, albeit in a slightly different manner. The non-local form of this action implies an important dependence on boundary conditions for the Green functions of the scalar field in the given background. We argue that the 
effective action only has a consistent interpretation when for that quantity the (otherwise unphysical) Boulware state determines the boundary condition at infinity (infrared regularisation). This is supported by a study of the Green functions where - in a formal perturbation series starting from flat spacetime - a natural boundary condition at the horizon directly leads to a constant homogeneous solution. The latter can be absorbed in a renormalisation constant which does not affect the asymptotic flux. Generically, the choice of different homogeneous solutions represents the ambiguity inherent in the non-local effective action.

From the regular part of that effective action in the Boulware state the Hawking flux is not derived directly, but only the non-radiative basic components $\langle T\rangle_{2}$ and $\left\langle T^{\theta}\right\rangle_{2}$ which are, indeed, independent of the assumed (Boulware, Hartle-Hawking, Unruh) quantum state [20. Inserting them into the EM (non-)conservation equation the correct Hawking flux in the Unruh state (in agreement with the black body derivation) is obtained, without proceeding through one mathematically questionable step which in refs. [13, 16] produced the same physically reasonable result.

We also clarify the consequences of the ambiguity brought about by different other choices of that homogeneous solution which are not constants. They may even yield a negative flux [10]. A choice like the one in that work, as noted by the authors themselves, violates the weak energy condition which we consider a more serious defect than the (logarithmic, hence integrable) divergence of some of the components of the EM tensor at the horizon [13, 16.

Beside the above-mentioned attractive features of the covariant perturbation theory, it reveals the existence of three new divergent terms (up to this order in curvature) which require further investigation. The first one $\propto c_{0}$ is interpreted as an infinite vacuum energy and its contribution to the flux can be removed by common renormalisation arguments. The second one $\propto c_{1}$ seems to be related to the boundary conditions as it contributes another logarithmic (UV) divergence at the horizon, as compared to the one produced by the homogeneous solution $\chi_{\rho}$, leaving a logarithmic IR divergence. Although we could show the independence (53) of the asymptotic flux of $c_{1}$ as well as of any constant $\chi_{\rho}$, the presence of an infinite constant in higher order terms in $r$ could not be avoided (at least, when renormalizing this constant to zero one is left with the logarithmic divergence at the horizon as in [13, 16]). Finally, our approach yields a completely new term (32) for the effective action that cannot be localized even in conformal gauge and contains an IR divergence $c_{2}$ which is coupled to an expression $\ln \square$. Although it could be shown that its contribution to the EM tensor can be given in a local form, the latter could not be evaluated further due to its ill-definedness. To sum it up, the 
covariant perturbation theory (by its non-local character) produced new IR divergent terms that were absent in the local Seeley-DeWitt expansion used in former derivations [13, 16. A proper renormalisation of these terms would require a detailed analysis of this method (e.g. considering higher orders in curvature or partial summations) which might be the issue of future work.

Beyond the application to the present problem the extension of covariant perturbation theory may set an example for the use of that technique in theories where matter is also coupled non-minimally to a scalar field (such as scalar-tensor theories). Also applications to a non-perturbative approach, where the geometry is integrated out, seem promising [22, 23].

The improved understanding of the situation for a static spherically symmetric Black Hole also seems a good basis for extending our result to include e.g. "grey factors" (ref. 50 in [8]) by admitting a non-vanishing classical scalar background field, back reaction upon the metric, and also the consideration of higher orders in the covariant perturbation series.

\section{Acknowledgement}

The authors thank especially D. V. Vassilevich (Leipzig) for clarifying discussions and D. Grumiller for a careful reading of the manuscript. This work was supported in part by the Austrian Science Foundation (FWF) Project 14.650-TPH which is gratefully acknowledged. 


\section{A Spherical Reduction}

The spherical reduction procedure is the basis of the two-dimensional dilaton model considered in this work. We reproduce it shortly, because some intermediate formulas are important for the main text. Here we consider a more general reduction from a $d$-dimensional spacetime $M$ with sphericallysymmetric metric

$$
d s^{2}=g_{\alpha \beta} d x^{\alpha} d x^{\beta}-\Phi^{2}\left(x^{\alpha}\right) g_{\kappa \lambda} d x^{\kappa} d x^{\lambda}
$$

to a two-dimensional Lorentz submanifold $L$, spanned by the coordinates $x^{\alpha}$ (e.g. $\left.t, r\right)$, by reducing out a $(d-2)$-sphere $S^{d-2}$ with coordinates $x^{\kappa}=$ $\theta, \varphi \ldots$ The dilaton field is defined as $X=\Phi^{2}, \Phi$ is more convenient for calculations. $g_{\alpha \beta}$ is the induced metric on the $L$ and $g_{\kappa \lambda}$ the one on $S^{d-2}$. We work in a vielbein basis in which the line-element can be written as

$$
d s^{2}=\eta_{a b} e^{a} \otimes e^{b}-\delta_{i j} e^{i} \otimes e^{j} .
$$

The $e^{r}$ form a vielbein basis on $M$. One can define a vielbein basis on $L$ and $S^{d-2}$ which we denote by $\tilde{e}^{a}$ and $\tilde{e}^{i}$, respectively ${ }^{11}$. They are related to the $e^{r}$ by

$$
\begin{aligned}
e^{a} & =\tilde{e}^{a}, e^{i}=\Phi \tilde{e}^{i} \\
E_{a} & =\tilde{E}_{a}, E_{i}=\Phi^{-1} \tilde{E}_{i} .
\end{aligned}
$$

Further, a (Levi-Civitá) spin-connection $\omega_{s}^{r}$ on $M$ induces connections on the submanifolds:

$$
\tilde{\omega}_{b}^{a}=\omega_{b}^{a}, \tilde{\omega}_{j}^{i}=\omega^{i}{ }_{j} .
$$

The connection on $M$, determined by vanishing torsion on $M$ and $L$, is given by

$$
\omega_{s}^{r}=\left(\begin{array}{cc}
\tilde{\omega}^{a}{ }_{b} & \left(\tilde{E}^{a} \Phi\right) \tilde{e}_{i} \\
\left(\tilde{E}_{a} \Phi\right) \tilde{e}^{i} & \tilde{\omega}^{i}{ }_{j}
\end{array}\right) .
$$

With (65) the Riemann tensor on $M$ can be expressed by geometrical objects on $L$ and $S^{d-2}$. For the scalar curvature one has

$$
\begin{aligned}
R^{M}=R^{L}- & \frac{1}{\Phi^{2}}\left[R^{S}+(d-2)(d-3)\left(\tilde{E}_{b} \Phi\right)\left(\tilde{E}^{b} \Phi\right)\right] \\
& -\frac{2}{\Phi}(d-2)\left[\left(\tilde{E}_{b} \tilde{E}^{b} \Phi\right)+\left(\tilde{E}^{b} \Phi\right) \tilde{\omega}_{b}^{a}\left(\tilde{E}_{a}\right)\right] \\
= & R^{L}-\frac{(d-2)(d-3)}{\Phi^{2}}\left[1+\tilde{\nabla}_{b} \Phi \tilde{\nabla}^{b} \Phi\right]-2\left(\frac{d-2}{\Phi}\right) \tilde{\square}_{L} \Phi .
\end{aligned}
$$

\footnotetext{
${ }^{11}$ We mark geometric objects belonging to $L$ or $S^{d-2}$ by a tilde; tensorial objects on the submanifolds are distinguished easily by the different indices used.
} 
In the last line we have inserted the (constant) scalar curvature of the $(d-2)$ sphere $R^{S}=(d-2)(d-3)$ (the Riemann tensor on the $(d-2)$-sphere is given by $\left.\tilde{R}_{j}^{i}=\tilde{e}^{i} \wedge \tilde{e}^{j}\right)$. In this form all quantities on the r.h.s. live on $L$ as it should be. By reduction from $d=4$ the Ricci tensor and the scalar curvature become

$$
\begin{aligned}
& R_{a b}^{M}=R_{a}\left(E_{b}\right)=R_{a b}^{L}-2 \frac{\tilde{\nabla}_{a} \tilde{\nabla}_{b} \Phi}{\Phi}=R_{a b}^{L}+\frac{\tilde{\nabla}_{a} X \tilde{\nabla}_{b} X}{2 X^{2}}-\frac{\tilde{\nabla}_{a} \tilde{\nabla}_{b} X}{X} \\
& R^{M}=R^{L}-2 \frac{1+(\tilde{\nabla} \Phi)^{2}}{\Phi^{2}}-4 \frac{\tilde{\square} \Phi}{\Phi}=R^{L}-\frac{2}{X}+\frac{(\tilde{\nabla} X)^{2}}{2 X^{2}}-2 \frac{\tilde{\square} X}{X} .
\end{aligned}
$$

In the last equality we have returned to the dilaton field $X=\Phi^{2}$. Note that the $\frac{\tilde{\square} X}{X}$-term in the scalar curvature becomes a surface term in the action when it is multiplied by the SR measure $\sqrt{-g_{M}}=X \sqrt{-g_{L}}$. For a d'Alembertian on the $d$-dimensional manifold $M$ acting on a scalar field $S\left(x^{\alpha}\right)$ which depends only on the coordinates of $L$ one gets

$$
\begin{aligned}
\square S=\eta^{r s} \nabla_{r} E_{s} S & =\eta^{a b} \nabla_{a} E_{b} S+\eta^{i j} \nabla_{i} E_{j} S \\
= & \tilde{\square} S-\eta^{i j} \omega^{a}{ }_{j}\left(E_{i}\right) E_{a} S=\tilde{\square} S-\eta^{i j} \frac{\tilde{E}^{a} \Phi}{\Phi} \tilde{E}_{a} S \\
& =\tilde{\square} S+(d-2) \frac{\tilde{\nabla}^{a} \Phi \tilde{\nabla}_{a} S}{\Phi}=\tilde{\square} S+\frac{d-2}{2} \frac{\tilde{\nabla}^{a} X \tilde{\nabla}_{a} S}{X} .
\end{aligned}
$$

If $M$ is the four-dimensional Schwarzschild spacetime and the gauge of the dilaton is fixed as $X=r^{2}$ this simplifies to

$$
\square S=\tilde{\square} S-\frac{2}{r}\left(1-\frac{2 M}{r}\right) \partial_{r} S .
$$

\section{B Green Function Perturbation - Higher Or- ders}

In this Appendix the second and third order of the Green function perturbation series (34) are adapted to the case of a 2d Schwarzschild spacetime with perturbing d'Alembertian (36), taking into consideration the particular boundary conditions on the half-plane and the time-independence of the involved integrals. The second order of the perturbation series then reads $\left(\partial^{\prime \prime}=\partial_{r^{\prime \prime}}\right)$

$$
-\int^{\prime \prime}\left[\partial^{\prime \prime} G_{0}\left(x, x^{\prime \prime}\right)\right] g\left(r^{\prime \prime}\right) \partial^{\prime \prime} G_{0}\left(x^{\prime \prime}, x^{\prime}\right) d^{2} x^{\prime \prime}
$$


where one $r^{\prime \prime}$-derivative has been partially integrated and

$$
g(r):=\frac{2 M}{r} .
$$

On a two-dimensional Schwarzschild spacetime $\partial_{r}^{2} g(r)=-R(r)$. A useful identity can be derived, introducing flat light-cone derivatives $\partial_{ \pm}=\partial_{t} \pm \partial_{r}$, $\square_{0}=\partial_{+} \partial_{-}=\partial_{-} \partial_{+}$. Because of the time-independence one can effectively set $\square_{0}=-\partial_{r}^{2}$ and $\partial_{+}=-\partial_{-}=\partial_{r},\left(g^{\prime \prime}=g\left(r^{\prime \prime}\right), G_{0}\left(x, x^{\prime}\right)=G_{x x^{\prime}}, \square_{0} G_{x x^{\prime}}=\right.$ $\left.-\delta^{2}\left(x-x^{\prime}\right)\right)$ :

$$
\begin{aligned}
0= & \int^{\prime \prime} \square_{0}^{\prime \prime}\left(G_{x x^{\prime \prime}} G_{x^{\prime \prime} x^{\prime}} g^{\prime \prime}\right) \\
& =-\left(g+g^{\prime}\right) G_{x x^{\prime}}-\int^{\prime \prime}\left\{\left(G_{x x^{\prime \prime}} G_{x^{\prime \prime} x^{\prime}}\right) R^{\prime \prime}+2 g^{\prime \prime}\left(\partial^{\prime \prime} G_{x x^{\prime \prime}}\right) \partial^{\prime \prime} G_{x^{\prime \prime} x^{\prime}}\right\}
\end{aligned}
$$

By (173) the second order of the perturbation series (34) can be written in the compact form

$$
\frac{1}{2}\left\{\left[g(r)+g\left(r^{\prime}\right)\right] G_{0}\left(x, x^{\prime}\right)+\int^{\prime \prime} G_{0}\left(x, x^{\prime \prime}\right) G_{0}\left(x^{\prime \prime}, x^{\prime}\right) R\left(r^{\prime \prime}\right) d^{2} x^{\prime \prime}\right\} .
$$

In a similar manner the third order is computed,

$$
\begin{gathered}
\int^{\prime \prime} \int^{\prime \prime \prime} G_{x x^{\prime \prime}} \partial^{\prime \prime}\left(g^{\prime \prime} \partial^{\prime \prime} G_{x^{\prime \prime} x^{\prime \prime \prime}}\right) \partial^{\prime \prime \prime}\left(g^{\prime \prime \prime} \partial^{\prime \prime \prime} G_{x^{\prime \prime \prime} x^{\prime}}\right) \\
=\int^{\prime \prime}\left(\partial^{\prime \prime} G_{x x^{\prime \prime}}\right) g^{\prime \prime} \partial^{\prime \prime} \int^{\prime \prime \prime}\left(\partial^{\prime \prime \prime} G_{x^{\prime \prime} x^{\prime \prime \prime}}\right) g^{\prime \prime \prime} \partial^{\prime \prime \prime} G_{x^{\prime \prime \prime} x^{\prime}} \\
=\frac{1}{4}\left\{\left[g^{2}+\left(g^{\prime}\right)^{2}\right] G_{x x^{\prime}}+\int^{\prime \prime} G_{x x^{\prime \prime}}\left[\partial^{\prime \prime}\left(g^{\prime \prime}\right)^{2}\right] \partial^{\prime \prime} G_{x^{\prime \prime} x^{\prime}}+\left[g^{\prime} g+\left(g^{\prime}\right)^{2}\right] G_{x x^{\prime}}\right. \\
\left.+g^{\prime} \int^{\prime \prime} G_{x x^{\prime \prime}} G_{x^{\prime \prime} x^{\prime}} R^{\prime \prime}+\int^{\prime \prime \prime} G_{x^{\prime \prime \prime} x^{\prime}} R^{\prime \prime \prime}\left(\left[g+g^{\prime \prime \prime}\right] G_{x x^{\prime \prime \prime}}+\int^{\prime \prime} G_{x x^{\prime \prime}} G_{x^{\prime \prime} x^{\prime \prime \prime}} R^{\prime \prime}\right)\right\},
\end{gathered}
$$


where again the identity (173) has been used. Finally, we write down the third order of the perturbation series in a compact form, replacing $\partial_{r} g^{2}(r)=2 R(r)$ :

$$
\begin{aligned}
& \frac{1}{4}\left\{\left[g^{2}(r)+g(r) g\left(r^{\prime}\right)+2 g^{2}\left(r^{\prime}\right)\right] G_{0}\left(x, x^{\prime}\right)\right. \\
& +\int^{\prime \prime} G_{0}\left(x, x^{\prime \prime}\right) R\left(r^{\prime \prime}\right)\left[2 \cdot \partial_{r^{\prime \prime}}+g\left(r^{\prime}\right)\right] G_{0}\left(x^{\prime \prime}, x^{\prime}\right) d^{2} x^{\prime \prime} \\
& +\int^{\prime \prime \prime} G_{0}\left(x^{\prime \prime \prime}, x^{\prime}\right) R\left(r^{\prime \prime \prime}\right) \\
& \left.\cdot\left(\left[g(r)+g\left(r^{\prime \prime \prime}\right)\right] G_{0}\left(x, x^{\prime \prime \prime}\right)+\int^{\prime \prime} G_{0}\left(x, x^{\prime \prime}\right) G_{0}\left(x^{\prime \prime}, x^{\prime \prime \prime}\right) R\left(r^{\prime \prime}\right) d^{2} x^{\prime \prime}\right) d^{2} x^{\prime \prime \prime}\right\} .
\end{aligned}
$$

\section{Non-Conservation Equation}

Diffeomorphism invariance of the generating functional implies a non-conservation equation for the renormalized expectation value of the EM tensor when a dilaton field is present, resulting from the non-minimal coupling of the scalar field in $d=2$ (cf. (2) ). A $2 d$ diffeomorphism transformation $\delta_{\xi} g^{\alpha \beta}=$ $-\left(\nabla^{\alpha} \xi^{\beta}+\nabla^{\beta} \xi^{\alpha}\right), \delta_{\xi} X=\xi^{\alpha} \partial_{\alpha} X, \delta_{\xi} S=\xi^{\alpha} \partial_{\alpha} S$ applied to the effective action $W[g]=-i \ln Z[g]$ yields the quantum non-conservation equation which has not been checked in the previous literature:

$$
\begin{aligned}
0= & \delta_{\xi} W[g]=\frac{-i \delta_{\xi} Z[g]}{Z[g]}=\frac{-i \mathcal{N}}{Z[g]} \delta_{\xi} \int \mathcal{D}(\sqrt[4]{-g} S) e^{i \int_{L} \frac{X}{2}\left[(\nabla S)^{2}-m^{2} S^{2}\right] \sqrt{-g} d^{2} x} \\
= & \frac{-i \mathcal{N}}{Z[g]} \int \mathcal{D}(\sqrt[4]{-g} S) \int_{L}^{y}\left[\delta_{\xi} g^{\alpha \beta} \frac{\delta}{\delta g^{\alpha \beta}}+\delta_{\xi} X \frac{\delta}{\delta X}+\delta_{\xi} S \frac{\delta}{\delta S}\right] e^{L_{d i l}^{m}} d^{2} y \\
= & \frac{-i \mathcal{N}}{Z[g]} \int \mathcal{D}(\sqrt[4]{-g} S) e^{L_{d i l}^{m}} \int_{L}^{y}\left\{-\left(\nabla^{\alpha} \xi^{\beta}+\nabla^{\beta} \xi^{\alpha}\right) i \frac{\sqrt{-g}}{2} T_{\alpha \beta}\right. \\
& \left.+\xi^{\alpha} \partial_{\alpha} X \frac{i \sqrt{-g}}{2}\left[(\nabla S)^{2}-m^{2} S^{2}\right]\right\} d^{2} y \\
& +\frac{i \mathcal{N}}{Z[g]} \int \mathcal{D}(\sqrt[4]{-g} S) e^{L_{d i l}^{m}} \int_{L}^{y} \frac{\delta}{\delta S} \delta_{\xi} S d^{2} y \\
= & \frac{\mathcal{N}}{Z[g]} \int \mathcal{D}(\sqrt[4]{-g} S) e^{L_{d i l}^{m}} \int_{L}^{y} \xi^{\alpha}\left\{\nabla^{\beta} T_{\alpha \beta}+\frac{\partial_{\alpha} X}{2}\left[(\nabla S)^{2}-m^{2} S^{2}\right]\right. \\
& \left.+\frac{i}{\sqrt{-g}} \lim _{x \rightarrow y} \partial_{\alpha} \delta(x-y)\right\} \sqrt{-g} d^{2} y \\
= & \int_{L}^{y} \xi^{\alpha}\left\{\nabla^{\beta}\left\langle T_{\alpha \beta}\right\rangle+\frac{\partial_{\alpha} X}{2}\left[\left\langle(\nabla S)^{2}-m^{2} S^{2}\right\rangle\right]\right. \\
& \left.+\frac{i}{\sqrt{-g}} \lim _{x \rightarrow y} \partial_{\alpha} \delta(x-y)\right\} \sqrt{-g} d^{2} y
\end{aligned}
$$


In the step from the second to the fifth line a partial integration in the path integral has been performed. The divergence $\delta(0)$ represents the infinite zero-point energy of the quantized scalar field $S$. Defining the renormalized EM tensor $\left\langle T_{\alpha \beta}\right\rangle_{r e n}:=\left\langle T_{\alpha \beta}\right\rangle+\frac{i g_{\alpha \beta}}{\sqrt{-g}} \lim _{x \rightarrow y} \delta(x-y)$ we obtain indeed (8) , as proposed.

\section{Heat Kernel Integrals}

The leading divergent $(T \rightarrow \infty, \varepsilon \rightarrow 0)$ terms of the expansions (19|23), appearing in the trace of the heat kernel (17) can be computed analytically. For instance (20) only has an UV divergence, hence we cutoff the $\tau$-integration at the lower boundary by $\varepsilon>0$. As a first step we expand the regularized integrand in powers of $s$, carry out the differentiation for $s$ and then set it zero:

$$
\begin{aligned}
&\left.\frac{d}{d s}\left\{\frac{1}{\Gamma(s)} \int_{\varepsilon}^{\infty} \tau^{s-1} d \tau\right\}\right|_{s=0} \\
&=\left.\frac{d}{d s}\left\{\left[s+s^{2} \gamma_{E}+O\left(s^{3}\right)\right] \int_{\varepsilon}^{\infty}\left[\tau^{-2}+s \tau^{-2} \ln \tau+O\left(s^{2}\right)\right] d \tau\right\}\right|_{s=0} \\
& \quad=\int_{\varepsilon}^{\infty} \tau^{-2} d \tau=\frac{1}{\varepsilon} .
\end{aligned}
$$

The most problematic term is (21). Introduction of a cutoff at infinity and differentiation for $s$ leads to (using (18):

$$
\begin{array}{r}
\left.\frac{d}{d s}\left\{\frac{1}{\Gamma(s)} \int_{0}^{T} \tau^{s} f(-\tau \triangle) d \tau\right\}\right|_{s=0}=\int_{0}^{1} \int_{0}^{T} e^{-\tau a(1-a)(-\triangle)} d \tau d a \\
=\int_{0}^{1} \frac{e^{-T a(1-a)(-\triangle)}-1}{a(1-a)(-\triangle)} d a=\frac{4}{-\triangle} \int_{0}^{1} \frac{e^{\left(z^{2}-1\right) \frac{T(-\triangle)}{4}}-1}{z^{2}-1} d z
\end{array}
$$

This integral cannot be solved analytically, however, it can be compared to

$$
\begin{aligned}
I=\int_{0}^{1} \int_{0}^{T} e^{(z-1) \frac{\tau(-\Delta)}{4}} d \tau d z=\frac{4}{-\triangle} \int_{0}^{1} \frac{e^{(z-1) \frac{T(-\Delta)}{4}}-1}{z-1} d z \\
=\frac{4}{-\triangle}\left\{\ln \left[\frac{T(-\triangle)}{4}\right]+\gamma_{E}\right\} .
\end{aligned}
$$

This is twice the result conjectured already at the r.h.s. of (21) apart from an additive constant $\frac{4 \ln 4}{\triangle}$. Hence, it remains to show that the difference between $I / 2$ and of the original expression (1.h.s. of (21) ) converges to (one 
half of) that constant for large regularisation parameter $T$. First we perform a substitution of the integration variable $z \rightarrow z^{2}$ in (80):

$$
\frac{I}{2}=\int_{0}^{T} \int_{0}^{1} z \cdot e^{\left(z^{2}-1\right) \frac{\tau(-\Delta)}{4}} d z d \tau
$$

This difference then becomes

$$
\begin{aligned}
\int_{0}^{1} \int_{0}^{T} e^{\left(z^{2}-1\right) \frac{\tau(-\Delta)}{4}}(z-1) d \tau d z & =\frac{4}{-\triangle} \int_{0}^{1} \frac{e^{\left(z^{2}-1\right) \frac{T(-\Delta)}{4}}-1}{z+1} d z \\
& \stackrel{\rightarrow \rightarrow \infty}{\rightarrow} \frac{4}{\triangle} \int_{0}^{1} \frac{1}{z+1} d z=\frac{4 \ln 2}{\triangle}=\frac{2 \ln 4}{\triangle}
\end{aligned}
$$

which proves (21) to be the correct result for large $T$. We note that the limit $\lim _{T \rightarrow \infty} e^{\left(z^{2}-1\right) \frac{T(-\Delta)}{4}}=0$ could be performed because $z^{2}<1$ for all $z$ except for $z=1$. But at that value the integrand vanishes altogether.

The computation of the remaining expressions is tedious but straightforward.

\section{References}

[1] S. W. Hawking. Commun. Math. Phys., 43:198, 1975.

[2] W. G. Unruh. Phys. Rev., D14:870, 1976.

[3] Bousso R and S. W. Hawking. Phys. Rev., D56:7788, 1997.

[4] P. Thomi, B. Isaak, and P. Hajicek. Phys. Rev., D30:1168, 1984.

[5] V. Mukhanov, A. Wipf, and A. Zelnikov. Phys. Let., B332:283-291, 1994.

[6] W. Kummer, H. Liebl, and D. V. Vassilevich. Mod. Phys. Lett., A12:2683, 1997, hep-th/9707041.

[7] W. Kummer, H. Liebl, and D. V. Vassilevich. Phys. Rev., D58:108501, 1998, hep-th/9801122.

[8] D. Grumiller, W. Kummer, and D. V. Vassilevich. Phys. Rept., 369:327429, 2002, hep-th/0204253.

[9] R. Balbinot and A. Fabbri. Phys. Rev., D59:044031, 1999, hep-th/9807123. 
[10] R. Balbinot and A. Fabbri. Phys. Lett., B459:112-118, 1999, gr-qc/9904034.

[11] F. C. Lombardo, F. D. Mazzitelli, and J. G. Russo. Phys. Rev., D59:064007, 1999, gr-qc/9808048.

[12] S. M. Christensen and S. A. Fulling. Phys. Rev., D15:2088-2104, 1977.

[13] W. Kummer and D. V. Vassilevich. Phys. Rev., D60:084021, 1998. hep-th/9811092.

[14] W. B. Gilkey. J. Diff. Geom., 10:601, 1975.

[15] M. E. Atiyah, V. K. Patodi, and I. M. Singer. Math. Proc. Camb. Phil. Soc., 79:985, 1976.

[16] W. Kummer and D. V. Vassilevich. Annalen Phys., 8:801-827, 1999, gr-qc/9907041.

[17] A. O. Barvinsky and G. A. Vilkovisky. Nucl. Phys., B282:163-188, 1987.

[18] A. O. Barvinsky and G. A. Vilkovisky. Nucl. Phys., B333:471-511, 1990.

[19] A. O. Barvinsky, Y. V. Gusev, G. A. Vilkovisky, and V. V. Zhytnikov. Nucl. Phys., B439:561-582, 1995, hep-th/9404187.

[20] D. Hofmann. Quantum Radiation from Black Holes. PhD thesis, Technische Universität Wien, 2002, gr-qc/0209007.

[21] K. Fujikawa, U. Lindström, N. K. Nielsen, M. Rocek, and P. van Nieuwenhuizen. Phys.Rev., D37:391, 1988.

[22] Daniel Grumiller. Quantum dilaton gravity in two dimensions with matter. PhD thesis, Technische Universität Wien, 2001, gr-qc/0105078.

[23] P. Fischer, D. Grumiller, W. Kummer, and D. V. Vassilevich. Phys. Lett., B521:357-363, 2001, gr-qc/0105034. Erratum ibid. B532 (2002) 373. 